\title{
SAMPEX Spin Stabilized Mode
}

\author{
Dean C. Tsai ${ }^{1}$ and F. Landis Markley ${ }^{2}$ \\ NASA Goddard Space Flight Center, Greenbelt, MD 20771 \\ Todd P. Watson ${ }^{3}$ \\ Honeywell Technology Solutions, Inc., Bowie, MD 20715
}

\begin{abstract}
The Solar, Anomalous, and Magnetospheric Particle Explorer (SAMPEX), the first of the Small Explorer series of spacecraft, was launched on July 3, 1992 into an $82^{\circ}$ inclination orbit with an apogee of $670 \mathrm{~km}$ and a perigee of $520 \mathrm{~km}$ and a mission lifetime goal of 3 years. After more than 15 years of continuous operation, the reaction wheel began to fail on August 18, 2007. With a set of three magnetic torquer bars being the only remaining attitude actuator, the SAMPEX recovery team decided to deviate from its original attitude control system design and put the spacecraft into a spin stabilized mode. The necessary operations had not been used for many years, which posed a challenge. However, on September 25, 2007 , the spacecraft was successfully spun up to $1.0 \mathrm{rpm}$ about its pitch axis, which points at the sun. This paper describes the diagnosis of the anomaly, the analysis of flight data, the simulation of the spacecraft dynamics, and the procedures used to recover the spacecraft to spin stabilized mode.
\end{abstract}

\section{Nomenclature}

$\begin{array}{ll}H_{0} & =\text { desired system angular momentum magnitude } \\ \mathbf{H} & =\text { total system angular momentum vector } \\ \Delta \mathbf{H} & =\text { undesired component of the system angular momentum vector } \\ \mathbf{H}_{w} & =\text { wheel momentum } \\ \mathbf{s} & =\text { sun vector } \\ \mathbf{j} & =\text { body pitch axis } \\ \hat{\mathbf{j}} & =\text { principal pitch axis } \\ \mathbf{b} & =\text { magnetic field vector } \\ \mathbf{I} & =\text { moment of inertia tensor } \\ \mathbf{\omega} & =\text { spin rate vector } \\ \mathbf{m} & =\text { commanded torquer dipole }\end{array}$

\section{Introduction}

$\mathrm{T}$

he Solar, Anomalous, and Magnetospheric Particle Explorer (SAMPEX), the first of the Small Explorer series of spacecraft, was launched on July 3, 1992 into an 82 degree inclination orbit with an apogee of $670 \mathrm{~km}$ and a perigee of $520 \mathrm{~km}^{1}$. The scientific purpose of SAMPEX is to study solar energetic particles, anomalous cosmic rays, magnetospheric relativistic precipitating electrons, and galactic cosmic rays ${ }^{1}$. The spacecraft is equipped with four instruments to carry out this mission: the Low Energy Ion Composition Analyzer (LEICA), the Heavy Ion Large Telescope (HILT), the Mass Spectrometer Telescope (MAST), and the Proton Electron Telescope (PET).

The attitude control system (ACS) uses a single reaction wheel and three orthogonal magnetic torquer bars to control the spacecraft attitude. After more than 15 years of continuous operation the reaction wheel began to fail on August 18, 2007. Upon the detection of the failure, the SAMPEX ground system notified the flight operations team about the anomaly through an automated mission monitoring and paging system. The spacecraft remained in the

\footnotetext{
${ }_{1}^{1}$ Aerospace Engineer, GN\&C Systems Engineering Branch, Code 591

${ }^{2}$ Aerospace Engineer, GN\&C Systems Engineering Branch, Code 591, AIAA Fellow

${ }^{3}$ Bowie State University Satellite Operations and Control Center (BSOCC) Program Lead
} 
science mode and continued to utilize the magnetic torquer bars to maintain sun point, but all the reaction wheel commands were being ignored. With the primary attitude actuator failing, SAMPEX can no longer rely on the attitude stability the wheel bias once provided. The spacecraft also became incapable of performing attitude control maneuvers for which the spacecraft was designed ${ }^{2,3}$. With inputs from the scientists, the SAMPEX operations and recovery team eventually decided to recover SAMPEX into a spin stabilized recovery mode by using the only remaining attitude actuator, the three magnetic torquer bars. This paper will give an overview of the SAMPEX mission, the original SAMPEX ACS Modes, and the operations team's effort to recover SAMPEX to the offnominal spin stabilized mode while still maintaining its scientific viability. SAMPEX successfully recovered to the spin-stabilized mode on September 25, 2008.

\section{Mission Overview}

The NASA Goddard Space Flight Center Small Explorer program (SMEX) was developed to provide frequent flight opportunities for highly focused and relatively inexpensive space science missions. The SMEX program recognized the benefits of small and quick turnaround projects, therefore the cost per mission was to be limited to $\$ 30$ million. An important aspect of SMEX was that one principal investigator would propose a complete mission and its experiments ${ }^{1}$. The idea was to have one team responsible for the entire mission and all the instruments. The results were highly efficient, highly cost effective research efforts in the areas of space physics, astrophysics, and upper atmospheric science. The Solar, Anomalous, and Magnetospheric Particle Explorer is the first mission of the SMEX program. The original mission lifetime was 3 years with the possibility of extension in order to study the 11 years solar cycle more extensively.

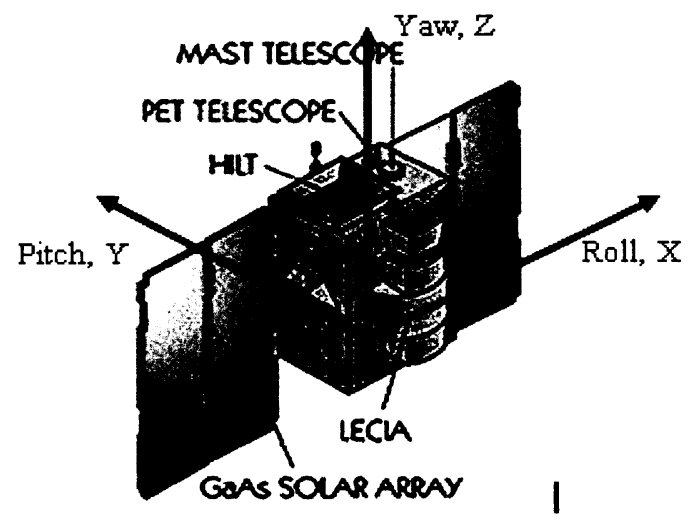

Figure 1. Configuration and Instruments. The solar arrays are fixed with their outward normals along the spacecraft $+y$ axis; power constraints require this axis to be sun-pointing at all times. The instrument fields-ofview all point along the spacecraft $+z$ axis.

The primary scientific objectives of SAMPEX are to measure the elemental and isotopic composition of solar energetic particles, anomalous cosmic rays, and galactic cosmic rays over the energy range from -1 to several hundreds of $\mathrm{MeV}$ per nucleon. By determining the dependence of the fluxes on geomagnetic cutoff rigidity over the polar orbit, the ionization state of the anomalous component is determined, along with the main ionization state of the solar energetic particles. The dependency of these fluxes on the solar activity cycle is measured by carrying out continuous observations over an extended portion of the current activity cycle. A further primary objective is to determine flux levels and local time dependence of relativistic precipitating magnetospheric electrons during a period of declining solar activity. The primary region of interest for these studies is over the north and south poles (latitude above $60 \mathrm{~N}$ and $60 \mathrm{~S}$ ). However, the instruments collect science data continuously throughout the orbit.

The original instrument pointing requirement was to point the z-axis as closely to the local zenith as possible in the polar regions, consistent with the sun-pointing requirement ${ }^{2-5}$. The original SAMPEX attitude control mode was the Orbit Rate Rotation (ORR) mode with the $y$-axis always sun-pointed and the $z$-axis rotating around the sunline at one revolution per orbit, synchronized such that the z-axis points as close to zenith as possible when the spacecraft reaches the northernmost and southernmost points in its orbit ${ }^{4,5}$. A later requirement, known as "velocity avoidance" was to avoid pointing the instrument boresights in the direction of spacecraft motion, to avoid damage by orbiting 
debris to delicate thin multiplayer entrance windows in the HILT instrument. An algorithm to accomplish this was incorporated into the ORR mode ${ }^{4}$.

Data taken from the few years of the SAMPEX mission confirmed the existence of a third radiation belt surrounding the Earth, in addition to the two Van Allen belts containing trapped electrons and protons, respectively ${ }^{6-8}$. The new belt, whose existence had been predicted 15 years earlier, contains trapped heavy ions, principally $\mathrm{O}, \mathrm{N}$, and $\mathrm{Ne}$. Analysis of the MAST data showed that the trapped particles are observed primarily when the instrument is viewing near 90 degrees to the local magnetic field line. With the ORR pointing mode, this viewing angle varies with a three-month period. In order to increase data collection on the trapped heavy ions, it was desirable to change the pointing algorithm to orient the instrument boresights perpendicular to the magnetic field line during every passage through the regions containing these particles.

The region of most interest is in the South Atlantic, and analysis of the magnetic field contours revealed that this region can be characterized as a region of low magnetic field strength ${ }^{9}$. In order to satisfy these revised science requirements, the SAMPEX pointing mode was modified using the strength of the magnetic field as a delimiter to point the spacecraft perpendicular to the magnetic field vector whenever the field is determined to be less than some specified value ${ }^{3}$. This pointing is accomplished by using magnetometer data to determine the field direction in the spacecraft reference frame. Since the magnetometer data was judged to provide a good pointing reference, the pointing specification was changed in the high-field regions to as close to the magnetic field vector as possible, consistent with the constraint that the $y$-axis be pointed at the sun. At northern latitude, the desired orientation is anti-parallel to the field, and in the south the orientation is parallel to the field. In both cases, then, the spacecraft points away from the earth in the polar regions. The ACS software that implements this was uploaded to the spacecraft in 1996, and it has been part of the ORR control mode ever since, including the time of the wheel anomaly.

\section{ACS Modes Overview}

The primary functions of the SAMPEX Attitude Control System are to acquire the sun after fourth stage separation from the launch vehicle, provide attitude control and determination during nominal flight operations, and supply an independent Safehold mode. The ACS stabilizes the spacecraft with bias momentum using a single momentum wheel whose spin axis is aligned with the sunline. A three-axis magnetometer, one two-axis fine sun sensor, and five coarse sun sensors are the primary sensing devices. The pitch momentum wheel and three magnetic torquer bars provide attitude control and momentum management. The reaction wheel angular momentum served to keep the pitch axis sun-pointing even when the sun is behind the Earth, so that full power was available when SAMPEX emerged from eclipse. The magnetic torquers were used to control the magnitude and direction of the system angular momentum when SAMPEX is in sunlight. Reaction wheel torque commands were used to control spacecraft rotation about the pitch axis. In case of an ACS contingency, an independent Safehold will orient the spacecraft indefinitely along the sunline. Major requirements that contributed to the SAMPEX ACS design:

1) Solar Arrays normal to sunline ( $+/-5 \mathrm{deg})$

2) Maximize instrument alignment with zenith over the poles $(>60 \mathrm{deg})$

3) Minimize instrument exposure to the velocity vector $(>80 \mathrm{deg})$

4) Independent Safehold mode

There are five major control modes and two science sub-modes residing in the ACS software. All major digital modes are entered via ground commands, and exited by either receipt of a ground command to a new pointing mode, or by an autonomous Safehold Triggering event. The sub-modes are autonomously entered by various triggering criteria, and they remain until the mode criteria are no longer true. There is also an independent analog Safehold mode used for saving the spacecraft in emergency conditions. A summary of each mode is given in the following section (Table 1).

\section{A. Science Pointing Modes}

These control modes null the output of the fine sun sensor, pointing the pitch axis at the sunline for maximum power. They use the on-board attitude estimate to control the wheel torque for rotation about the pitch axis. The only difference between the science pointing modes is the computation of the pitch angle error that is used to control the wheel torque, as described in the Mission Overview section above.

\section{B. Science Sub-Modes}

There are two sub-modes that are invoked autonomously onboard the spacecraft when the control system detects specific events. The modes are exited when the event condition is no longer satisfied or a Safehold trigger is 
detected (in which case the spacecraft automatically enters the analog Safehold mode). Control is automatically returned to the previous science mode. Since entry and exit of these sub-modes occurs automatically, no ground commands are provided to manually enter or exit these sub-modes. The sub-modes are only used when the control system is using a science control mode.

1. Coast Sub-Mode

The Coast Sub-Mode suspends magnetic actuation and holds the wheel speed at its most recent value. It is used to drift through mathematical singularities in the attitude determination solution when the magnetic field vector and sunline are co-aligned within 5 degrees during sun and with 40 degrees during eclipse. The vector coalignment is a function of orbital season and may happen as much as 4 times per orbit when the sunline lies in or near the orbit plane. The duration of the coalignment is small. Spacecraft drift during coalignment does not degrade pointing performance; however no onboard attitude determination is performed in this mode. When the ACS detects that the two vectors are no longer with 5 degrees of each other, the controller returns to the previous science pointing mode.

\section{Eclipse Sub-Mode}

The Eclipse Sub-Mode is enabled automatically during a science pointing mode when the sun presence flag returns a value of false. It suspends magnetic actuation and determines the sun vector from the assumption that the spacecraft pitch axis (wheel momentum vector) remains initially fixed. For short periods of time ( $<1$ hour) this assumption is reasonable. This process allows three-axis attitude determination to within the 2 degree requirement when the sun is eclipsed. Attitude determination is then performed normally with the measured magnetic field vector and derived sun vector. The pitch error is computed and the appropriate torque is commanded to the momentum wheel. When the sun presence flag returns to true, the controller returns to the previous science pointing mode.

Table 1. ACS Modes and Sub-Modes

\begin{tabular}{|c|c|c|c|c|}
\hline Control Mode & Purpose & Enter & Exit & Duration \\
\hline Orbit Rotation Rate & Science & $\begin{array}{c}\text { Ground Command or Return } \\
\text { From Sub-mode }\end{array}$ & $\begin{array}{c}\text { Ground Command, Sub-mode } \\
\text { Trigger, or Analog Safehold }\end{array}$ & - \\
\hline Vertical Pointing & Science & $\begin{array}{c}\text { Ground Command or Return } \\
\text { from Sub-mode }\end{array}$ & $\begin{array}{c}\text { Ground Command, Sub-mode } \\
\text { Trigger, or Analog Safehold }\end{array}$ & - \\
\hline Coast Sub-mode & $\begin{array}{c}\text { Co-alignment of } \\
\text { Sun and Magnetic } \\
\text { Field Vectors }\end{array}$ & $\begin{array}{c}\text { Automatic - within 5 degrees } \\
\text { of sun; within 40 degrees in } \\
\text { eclipse }\end{array}$ & $\begin{array}{c}\text { Automatic - when triggering } \\
\text { criteria no longer applies, } \\
\text { Analog Safehold }\end{array}$ & Five minutes, worst case \\
\hline Sun Point Only & Eclipse & $\begin{array}{c}\text { Automatic - Loss of Sun } \\
\text { pulse signal }\end{array}$ & $\begin{array}{c}\text { Automatic - Return of Sun } \\
\text { pulse signal, Analog Safehold }\end{array}$ & $\begin{array}{c}\text { Up to 35 minutes per } \\
\text { orbit in shadow seasons }\end{array}$ \\
\hline $\begin{array}{c}\text { Magnetometer } \\
\text { Calibration }\end{array}$ & $\begin{array}{c}\text { Software } \\
\text { Safehentation of } \\
\text { Calibration }\end{array}$ & $\begin{array}{c}\text { Ground Command or } \\
\text { whenever Roll, Yaw Error } \\
\text { greater than 30 degrees }\end{array}$ & $\begin{array}{c}\text { Ground Command, whenever } \\
\text { Roll, Yaw Error less than 30 } \\
\text { degrees, or Analog Safehold }\end{array}$ & - \\
\hline Manual Override & $\begin{array}{c}\text { Ground Control of } \\
\text { Actuators }\end{array}$ & Ground Command & $\begin{array}{c}\text { Automatic - returns to previous } \\
\text { control mode, Analog Safehold }\end{array}$ & Four seconds \\
\hline
\end{tabular}

\section{Other Modes}

\section{Magnetometer Calibration}

The Magnetometer Calibration Mode is used to sense the unwanted component of the signal on the magnetometer produced by the $\mathrm{x}, \mathrm{y}$, and $\mathrm{z}$-axis torquer bars and adjust the coupling matrix values that remove the contamination. This mode takes approximately 4 seconds to execute. Prior to launch, initial values are loaded into the coupling matrix based on results from spacecraft magnetic calibration testes. This mode was designed to use once initially to correct the initial values of the coupling matrix and then periodically update to account for any long term changes in the magnetic properties of the spacecraft. In practice, the operations team only performs Magnetometer Calibration upon return from the analog Safehold.

\section{Sun Point Only}

This mode is a digital mode equivalent to the Analog Safehold Mode. Although three-axis attitude determination is performed, control is only attempted in the roll and yaw axes. It is not meant to be part of the nominal spacecraft operations, therefore it can only be entered through ground commanding. The justification for including this additional mode is that the digital logic affords the flexibility of changing control parameters that are fixed in the analog Safehold electronics. Any modification to this control mode can be accomplished in the form of a memory patch. It also acts as a buffer between digital control modes and the analog Safehold. It has been a standard 
operations practice to command the spacecraft to this mode upon exit of Safehold, this allows a diagnostic of the digital controller functionalities before transitioning to an actual science mode.

\section{Manual Overrides}

In addition to all the digital control modes listed above, there are manual override commands for each of the spacecraft actuators ( $\mathrm{x}, \mathrm{y}$, and $\mathrm{z}$-axis torquer bars and momentum wheel). Not a fully independent mode, the override logic computes the appropriate torquer bars current or wheel torque and replaces that specific actuator command with the override commanded value. The actuators that are not overridden are commanded by the current digital pointing mode. The manual override command format is an enable/disable flag and a level. The commanded level is given in dipole moment for the torquer bars and wheel speed for the momentum wheel. The override command lasts for the specified number of dwell cycles, after which an additional override command must be issued to continue manual control of the actuator, if desired. The exiting condition is either by an equivalent disable command or by the detection of a Safehold triggering event.

\section{Independent Analog Safehold}

The analog Safehold mode is a set of independent analog electronics in the Attitude Control Electronics box (ACE). The system is fully independent in the sense that as long as the ACE has power, the Safehold Mode will operate and provide commands to the actuators. During normal operations, the actuator drives receive two separate groups of actuator commands: 1) from the Analog Safehold Mode and 2) from the current active Digital control Mode or Sub-mode. The Analog Safehold Electronics selects the appropriate actuator command set based on the status of three events: 1) whether a power reset indicator has been cleared, 2) whether a Safehold On Command has been received, and 3) whether the Safehold Pulse (Me-Ok) signal from the Digital Control Mode has timed out.

Additionally, there are eight bi-level commandable switches which allow for several possible configuration of the analog Safehold mode. For initial sun acquisition after launch and under certain conditions a default switch setting is automatically selected for Safehold configuration. These switches and their possible settings are detailed in Table 2.

Table 2. Analog Safehold Switches

\begin{tabular}{|l|l|l|l|}
\hline Switch & Settings & Default & Description \\
\hline $\begin{array}{l}\text { 1. X-axis spin/de-spin } \\
\text { 2. Z-axis spin/de-spin }\end{array}$ & $\begin{array}{l}\text { Spin/De-spin } \\
\text { Spin/De-spin }\end{array}$ & $\begin{array}{l}\text { Spin } \\
\text { Spin }\end{array}$ & $\begin{array}{l}\text { Configures specified axis for spinning the S/C in one direction or the other when } \\
\text { commanding the specified torquer bar continuously. These switches are set as a pair } \\
\text { (both either spin or de-spin). }\end{array}$ \\
\hline $\begin{array}{l}\text { 3. X-axis connect } \\
\text { 4. Z-axis connect }\end{array}$ & $\begin{array}{l}\text { On/Off } \\
\text { On/Off }\end{array}$ & $\begin{array}{l}\text { Off } \\
\text { Off }\end{array}$ & $\begin{array}{l}\text { Individually commandable; connects selected torquer bar full-on. Disabled when switch } \\
\text { is in the off position }\end{array}$ \\
\hline $\begin{array}{l}\text { 5. Beta-Dot on X-axis } \\
\text { 6. Beta-Dot on Y-axis } \\
\text { 7. Beta-Dot on Z-axis }\end{array}$ & $\begin{array}{l}\text { On/Off } \\
\text { On/Off }\end{array}$ & On & Individually commandable; enables/disables Beta-Dot for each axis. \\
\hline 8. Sun Point & On/Off & On & Used for Y-axis control; enables/disables sun point mode. \\
\hline
\end{tabular}

A. Safehold Triggering Events

There are three events which will result in transition to the Analog Safehold Mode.

\section{Power Reset}

The Power Reset Latch could be set, intentionally or unintentionally, indicating a power interruption, the spacecraft will enter the analog Safehold mode. All the switches will return to their default settings.

\section{Safehold On ground command received}

A Safehold On/Off switch is provided which may be changed by the Switch Set command packet received from the ground. This command goes directly to the ACE Box via the 1773 bus bypassing the ACS software. All the switches remain at their present settings as the spacecraft transitions to analog Safehold. They can be re-set with another Switch Set command packer during Safehold. Once the SH ON flag is set, the spacecraft remains in Analog Safehold Mode until a SH Off ground command is received.

3. Loss of Safehold Pulse (Me-OK) signal

The Safehold Pulse signal is used to reset a counter in the Analog Safehold electronics. If no Safehold Pulse signal is received in 10 cycles ( 5 seconds) from the ACS software, the circuit times out and invokes Analog Safehold Mode. Loss of the Safehold Pulse may occur from two types of events: operational failure or the suspension of Safehold Pulse in the ACS software. An operational failure could be produced by a hardware failure, such as a bus failure, or by a software failure such as a timing problem, scheduler failure, or ACS software failure. In all cases, operational failures are unanticipated interruptions of the active digital control mode and transition to Analog Safehold Mode is considered necessary to preserve the spacecraft health. There are three things that could trigger the ACS software to issue the suspension of the Safehold Pulse: Small Explore Data System (SEDS) cold 
reboot, Loss of sun lock (pitch axis within 30 degrees from the sunline), and receipt of Safehold Pulse Disable ground command. In all three cases, the spacecraft remains in Analog Safehold Mode until the Safehold Pulse Enable Ground command is received.

\section{Nominal Operations}

In January 1997, SAMPEX mission operations was transferred from NASA's Goddard Space Flight Center to the Bowie State University Satellite Operations and Control Center (BSOCC) in Bowie, Maryland, where flight operations are still performed. The BSOCC is a student flight control training facility supported by NASA, Bowie State University and Honeywell Technology Solutions, Inc. The control center is home to flight operations for both SAMPEX and NASA's Wide-field Infrared Explorer (WIRE). The students in the program are able to earn three progressively advanced levels of mission control certification while taking part in hands-on operations under the guidance of a Honeywell professional.

With the help of automated systems, BSOCC monitors and controls the missions in a normal Monday-Friday daytime work week. During nights and weekends the flight control team is notified of anomalous developments through an automated paging system. As mentioned earlier, this is how the FOT was first alerted to the reaction wheel failure on Saturday, August 18, 2007. In a typical week, several command loads are uplinked to each spacecraft, which include stored commands for transmitting scientific and health and safety data to the ground on each of the two to three earth station contacts per day. The health and safety data is analyzed and archived in BSOCC, and the scientific data is processed and delivered to the science teams.

The initial phase of SAMPEX science data collection ended in July, 2004. During this period the principal investigator was Glenn Mason of the University of Maryland's Department of Physics, with co-investigators located at the Max Planck Institute, California Institute of Technology and the Aerospace Corporation of El Segundo, California. In August, 2004, Joseph Mazur of the Aerospace Corporation took over the SAMPEX experiment for space weather research, and it continues to perform space weather analysis up to now.

\section{The Reaction Wheel Failure Anomaly}

On August 18, 2007, the FOT was notified at home through the automated mission monitoring and paging system about an anomaly with the SAMPEX spacecraft's attitude control system. The team immediately reported back to the Bowie State control center and prepared for on-console diagnostics during the next real-time pass opportunity. During the real-time contact, the FOT discovered several off-nominal indicators: the reaction wheel had been running hot and was spinning at significantly lower speed than required for the momentum bias design. The attitude determination system estimated the spacecraft body rate had increased from one revolution per orbit about the pitch axis to about one revolution for every two and a half minutes, an indication that most of the angular momentum of the wheel had been transferred to the spacecraft itself. Fortunately, other than the anomalous state of the reaction wheel, there were no other signs of failures. The spacecraft maintained in its pre-failure science ORR mode, and because of the law of conservation of momentum, the spacecraft appeared to be spin stabilized at the time. Reaction wheel commands sent from the ACS were simply ignored. In the subsequent passes, the FOT tried to revive the reaction wheel through power cycling and sending it both negative and positive spin commands, but the attempts were not effective. The FOT further reviewed the Health and Safety data, but it did not provide enough insight to determine the root cause of the reaction wheel failure.

While the FOT was evaluating the impact of the wheel failure and its ramifications for the safety of the spacecraft, the science counterparts at the Aerospace Corporation were asked to evaluate the impact of the $0.4 \mathrm{rpm}$ spin-rate on the science instruments. As the FOT concluded that the reaction wheel failure was not recoverable, the scientists arrived at the conclusion that the current spin rate was not detrimental to the science instruments. They further concluded that even though the quality of the science data was degraded, they were still usable. The scientists stated a preference for SAMPEX to increase its spin rate from $0.4 \mathrm{rpm}$ to $1.0 \mathrm{rpm}$ to establish continuity with data gathered on several occasions prior to January 2000 when SAMPEX had been placed into a $1.0 \mathrm{rpm}$ spinning state to interrogate the heavy ions in the magnetosphere during solar maximum. The difference between then and now was that the momentum bias the wheel once provided is no longer present. So with the reaction wheel failure, it became apparent to the scientists that SAMPEX can no longer perform the Mid Latitude Modified Pointing Maneuver (Special Pointing Mode ${ }^{3}$ ). And the best they can hope for is for SAMPEX to maintain sun pointing while taking more science data. From an ACS standpoint, the higher spin-rate provides higher system stability and is thereby less susceptible to attitude disturbance and drift. Since the failed wheel simply rejected all wheel commands, no software update to the ACS modes was necessary. The challenge became configuring the damaged SAMPEX spacecraft to an attitude state that was not part of the original design. 


\section{Recovery Methodology}

To address the steps leading to recovery, it would be helpful to first elaborate on the magnetic control algorithm. When SAMPEX is in sunlight, its attitude is estimated using the TRIAD algorithm, with the Earth's magnetic field and the sun vector as references. The total spacecraft angular momentum, $\mathbf{H}$, is computed as the sum of the body angular momentum and the reaction wheel angular momentum, where the spacecraft angular velocity is obtained by differentiating the attitude matrix.

$$
\mathbf{H}=\mathrm{I} \boldsymbol{\omega}+\mathbf{H}_{w}
$$

The desired angular momentum is directed along the pitch axis (stable axis) and along the sunline (to keep the solar arrays sun-pointing) and has magnitude specified by a database value $\mathrm{H}_{0}$. Thus an undesired component of spacecraft angular momentum $\Delta \mathbf{H}$ is computed as

$$
\Delta \mathbf{H}=\left(\mathbf{H}-H_{0} \mathbf{j}\right)+\left(\mathbf{H}-H_{0} \mathbf{s}\right)=2 \mathbf{H}-H_{0}(\mathbf{j}+\mathbf{s}),
$$

where $\mathbf{j}$ and $\mathbf{s}$ are pitch axis and the sun vector respectively. The magnetic torquers are commanded to have dipole moment

$$
\mathbf{m}=k_{\text {mag }} \Delta \mathbf{H} \times \mathbf{b}
$$

where $\mathbf{k}_{\text {mag }}$ is a constant gain and $\mathbf{b}$ is the magnetic field vector measured in the spacecraft frame. The magnetic control is turned off when no reliable attitude estimate is available, which is during eclipse and also when the magnetic field vector is within $5^{\circ}$ of the sun vector.

About two weeks after the initial reaction wheel anomaly was discovered, the scientists proposed to spin up the spacecraft to $1.0 \mathrm{rpm}$. The value of $\mathrm{H}_{\mathrm{o}}$ used during most of the SAMPEX operations was $0.813 \mathrm{Nms}$ (Eq.1) When the reaction wheel failed, all the angular momentum stored in the wheel subsequently transferred into the spacecraft body resulted in a spin rate of $0.4 \mathrm{rpm}$. Raising the spin rate to $1.0 \mathrm{rpm}$ could be accomplished by increasing $\mathrm{H}_{\mathrm{o}}$ to $2.033 \mathrm{Nms}$. The anomaly resolution team quickly assembled a full-up spacecraft simulation that closely mimics the Orbit Rate Rotation mode.

A simulation of the spin-up maneuver demonstrated that a successful spin up concept would work with the existing controller logic (Fig.2). The simulation results showed the spacecraft gracefully spins up from $0.4 \mathrm{rpm}$ to $1.0 \mathrm{rpm}$ about its pitch-axis in about 60 minutes. The spin rate about the roll and yaw-axes are nonzero because there is a slight 3.5 deg angular offset between spacecraft body axes and the principal axes. As a result, the ACS utilizes the magnetic torque bars to spin up the spacecraft and pull the body pitch axis towards the sun vector at the same time. But since the pitch axis is not the principal spin-axis, the spin-rate about the spacecraft roll and yaw axes are non-zero. Figure 3 shows the torquers working hard trying to keep the principal axes aligned with the body axes. During the eclipse, the ACS transitions into the Coast Sub-Mode where the control actuation is temporarily turned off allowing the spacecraft to free drift. The angular momentum oscillation seen in the body frame about the roll and yaw axis during the eclipse is a natural motion known as coning, which is when the spacecraft freely spins about its principal spin axis.

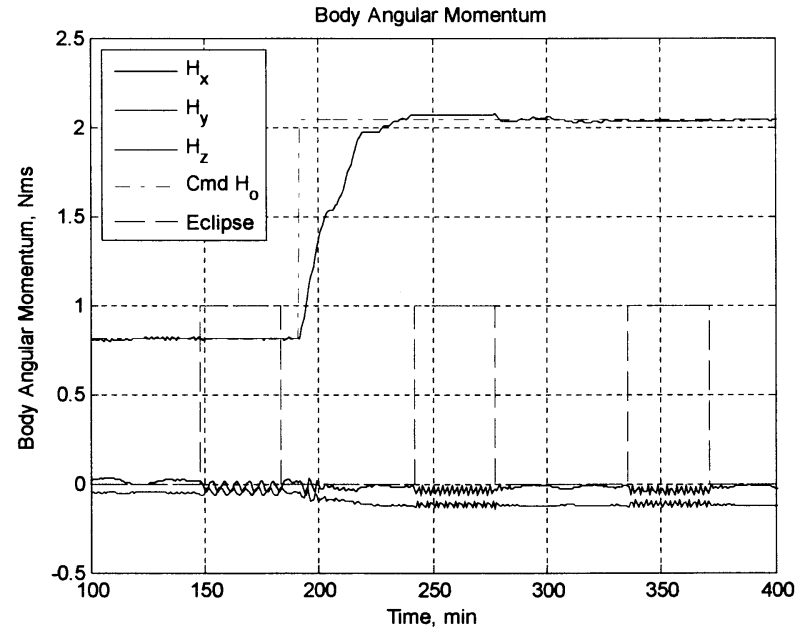

Figure 2. Simulated Angular Momentum of the System 


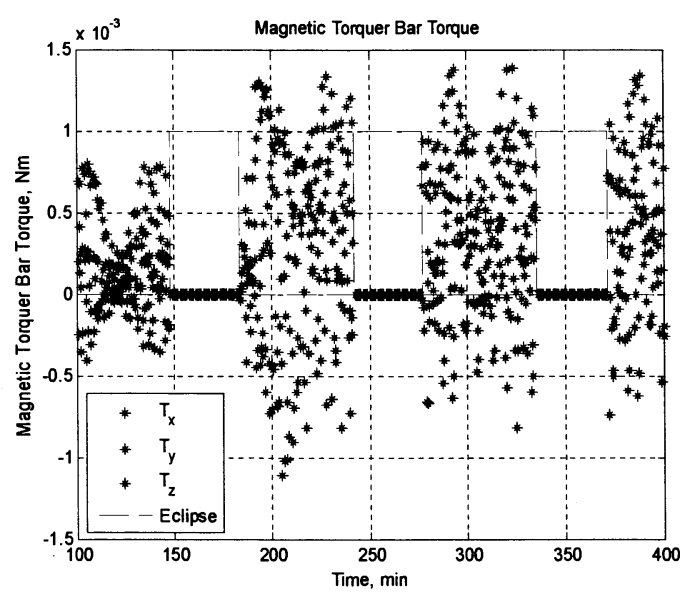

Figure 3. Simulated Torquer Bars Output Torque. The magnetic torquer bars continuously torque the spacecraft during the sunlit portion of the orbit to keep the spacecraft spinning about the body $z$ axis.

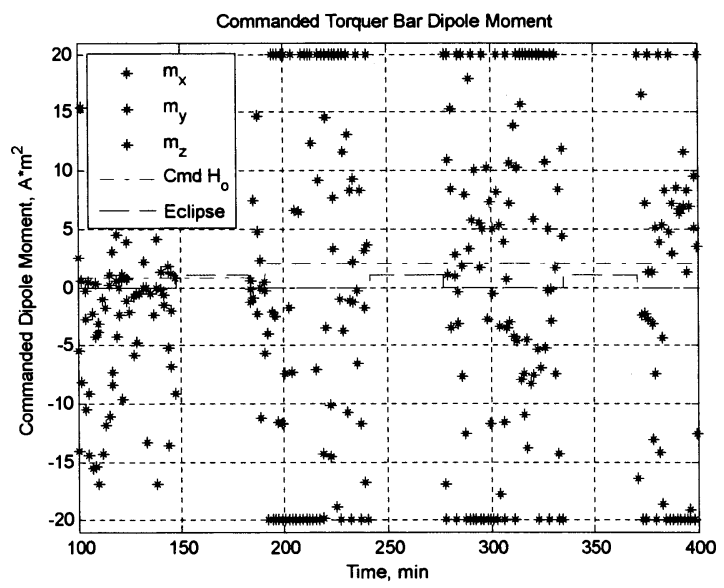

Figure 4. Simulated Commanded Dipole Moment. The torquers are saturated against their design limits with the new commanded $H_{0}$.

Figure 4 shows the torquer bars commanded dipole moment. During the sunlit portion of the orbit, the torquer bars often saturate their dipole moment limit, $+/-20 \mathrm{Am}^{2}$, in attempting to provide the desired control torque. This posed a minor concern for the health and safety of the magnetic torquer bars, the only ACS actuator left on SAMPEX. After consultation with the ACS hardware experts, it was concluded that the $+/-20 \mathrm{Am}^{2}$ was merely a software design limit, and that the torquer bars themselves can endure much higher dipole moment. The assessment came as a relief to the SAMPEX team, because the alternative would be a flight software update on the ACS controller to stop the ACS from overexerting the magnetic torquer bars. The momentum error would need to be modified to

$$
\Delta \mathbf{H}=\left(\mathbf{H}-H_{0} \hat{\mathbf{j}}\right)+\left(\mathbf{H}-H_{0} \mathbf{s}\right)=2 \mathbf{H}-H_{0}(\hat{\mathbf{j}}+\mathbf{s}),
$$

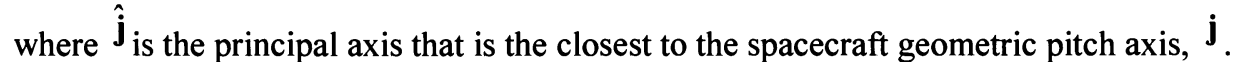

There was also a concern that if the spin-up maneuver were to be initiated at an inappropriate time, the sun error angle, from the pitch axis, could violate the default limit, and entry into Safehold would occur. An inappropriate time would be when all three torquer bars are used to initiate the spin up maneuver. This would occur whenever the sun vector and magnetic field vector were not nearly perpendicular. A nutation would be induced, thus increasing the sun error angle, possibly to the point of Safehold entry. The level of nutation can be significantly reduced if the timing of the maneuver could be limited to when the sun vector and the magnetic field vector were near perpendicular. At this time only the y-torque would be used to initiate the spin. This is optimal since spin along the pitch axis is the intent of the maneuver anyways. This desired situation occurs four times per orbit, twice at equator crossings, once as the spacecraft enters eclipse, and once as the spacecraft enters sunlight. Sunlight entry was chosen as the best starting point, since the system would have an entire sun period to spin the spacecraft up to the desired $1.0 \mathrm{rpm}$.

Simulation results showed that as long as the maneuver occurs just after the spacecraft has entered sunlight, the sun error angle is roughly $20 \mathrm{deg}$ at its largest (Fig.5), compared to $30 \mathrm{deg}$ threshold required for spacecraft transition to Safehold. As a cautionary measure, the FOT team decided to raise the threshold to 60 deg anyway to prevent Safehold from interrupting the spin-up maneuver. 
As another precaution against unexpected entry to Analog Safehold Mode during recovery, the FOT included two commands to switch the Safehold 'Beta-Dot on X-axis' and the 'Beta-Dot on Z-axis' switches from their default ON position to OFF, see Table 2. In the original SAMPEX three-axes stabilized with wheel momentum bias ACS design, the Safehold mode maintains the wheel at a constant speed and relies solely on the beta-dot controls of the torquers to stabilize the spacecraft body itself against undesirable rates on all three axes. In this new wheel-absent spacecraft configuration, should the spacecraft transition into Safehold, the beta-dot controls active on the body $\mathrm{x}$ and $\mathrm{z}$ axes would cause the spacecraft to de-spin about the pitch axis, which would effectively destabilize the spacecraft.

Figure 6 and Figure 7 are the actual system momentum and the sun angle measurement from flight data. The maneuver started around Day 268 16:32:00. As shown by the system momentum, the spacecraft successfully reached the destination in about 40 minutes, but it took about another 30 minutes to fully settle. The sun angle measurement confirmed that the spin-up maneuver had caused a slight attitude perturbation about the pitch axis, but it was not near the original $30 \mathrm{deg}$ threshold for triggering Safehold. There are slight deviations between the simulated and the actual flight data on the $\mathrm{x}$-axis and z-axis angular momentum and the sun angle. The former is attributed to model imperfections due to limited knowledge about the true inertia tensor. The latter is largely an effect of approximating spacecraft ephemeris for the time of the maneuver. Both approximations were known to the operations and recovery team, but they were secondary effects that would not affect the main recovery concept.

\section{Off-Nominal Operations and Science}

After the spin up procedure was completed, the $1.0 \mathrm{rpm}$ spin configuration requires little intervention from the ground controllers. During periods of $1.0 \mathrm{rpm}$ spin in previous years, the reaction wheel speed had to be held constant through a series of ground initiated commands. Otherwise, the wheel would become overtaxed as it would begin to absorb momentum from spacecraft. In cases of 1.0 RPM spin (both before and after the wheel failure), the amount of ACS telemetry generated increases to the point that the onboard recorder would quickly overfill. This problem is solved through uplinking a data storage filter table that slows down the data sampling rate.

From the science perspective, prior analysis of the PET and HILT instrument response to electrons had revealed gaps in scientists' knowledge of the response of the detectors to electrons incident at various angles of incidence.

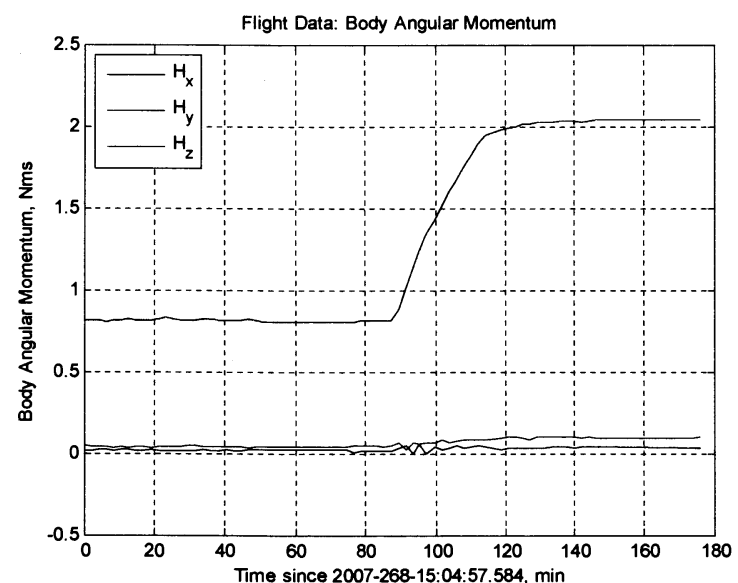

Figure 6: System Momentum during the Maneuver

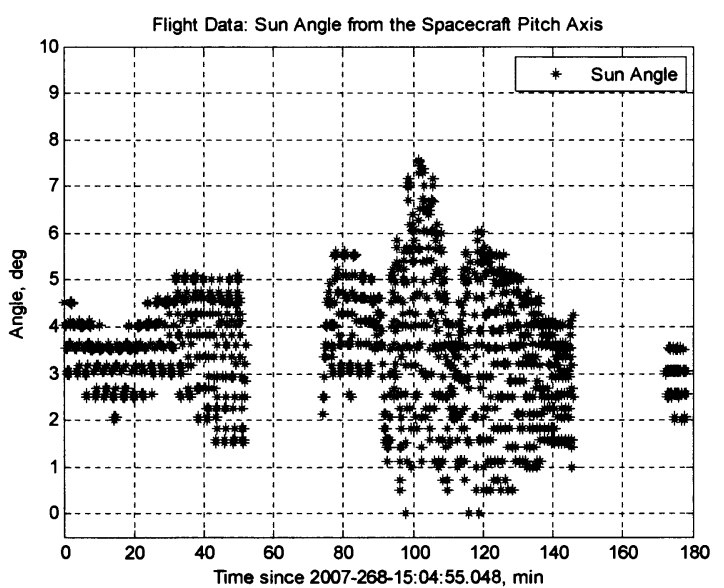

Figure 7. Sun Angle during the Maneuver 
Spinning motion about the pitch axis allows the two sensors to interrogate their vicinity with changing angular incidence of the incoming electron beam. Though spin stabilized mode was not part of the original design of the SAMPEX mission, it is still providing valuable science data.

\section{Conclusion}

The successful and timely recovery of the SAMPEX spacecraft from the failure of a key hardware component demonstrates the ability of the SAMPEX project and of the NASA Goddard Space Flight Center to diagnose and resolve flight anomalies effectively. This achievement is a result of a robust onboard control system, an effective operations design and execution, including the automated paging system for notifying operations personnel. In addition, close collaboration among Goddard Space Flight Center, the Bowie State University Satellite Operations and Control Center and the science team, as will as access to the original design personnel and documentation were invaluable.

\section{References}

${ }^{1}$ D. N. Baker, G. M. Mason, O. Figueroa, G. Colon, J. G. Watzin, and R. M. Aleman, "An Overview of the Solar, Anomalous, and Magnetospheric Particle Explorer (SAMPEX) Mission," IEEE Transactions on Geoscience and Remote Sensing, Vol. 31, No. 3, pp. 531-541, May 1993

${ }^{2}$ Thomas W. Flatley, Josephine K. Forden, Debra A. Henretty, E. Glenn Lightsey, and F. Landis Markley, "On-board Attitude Determination and Control Algorithms for SAMPEX," NASA/GSFC Flight Mechanics/Estimation Theory Symposium, Greenbelt, MD, May 22-24, 1990

${ }^{3}$ F. Landis Markley, Thomas W. Flatley, and Theodore Leoutsakos, "SAMPEX Special Pointing Mode," NASA/GSFC Flight Mechanics/Estimation Theory Symposium, Greenbelt, MD, 1995.

${ }^{4}$ Joseph P. Frakes, Thomas W. Flatley, Josephine K. San, Debra A. Henretty, F. Landis Markley, and E. Glenn Lightsey, "SAMPEX Science Pointing with Velocity Avoidance," AAS/AIAA Spaceflight Mechanics Meeting, Colorado Springs, CO, February 24-26, 1992

${ }^{5}$ Jon D. McCullough, Thomas W. Flatley, Debra A. Henretty, F. Landis Markley, and Josephine K. San, "Testing of the Onboard Attitude Determination and Control Algorithms for SAMPEX," NASA/GSFC Flight Mechanics/Estimation Theory Symposium, Greenbelt, MD, May 5-7, 1992

${ }^{6}$ Douglas Birch, "Earth Has Third Zone of Radiation,” Baltimore Sun, Vol. 313, No. 9, p.16A, May 26, 1993.

${ }^{7}$ Vincent Kiernan, "Small Explorer Satellite Finds Radiation Belt," Space News, March 31-June, 1993.

${ }^{8}$ J. R. Cummings, A. C. Cummings, R. A. Mewaldt, R. S. Selesnick, E. C. Stone, and T. T. von Rosenvinge, "New Evidence for Geomagnetically Trapped Anomalous Cosmic Rays." Geophysical Research Letters, Vol. 20, No. 18, pp. 2003-2006, September 15, 1993.

${ }^{9}$ E. G. Stassinopoulos, "World Maps of Constant B, L, and Flux Contours," NASA SP-3054, Goddard Space Flight Center, 1970 